Check for updates

Cite this: RSC Adv., 2017, 7, 33078

\section{Moderate oxidation levels of Ru nanoparticles enhance molecular oxygen activation for cross- dehydrogenative-coupling reactions via single electron transfer $\dagger$}

\author{
Mu Lin,,$^{a}$ Lin-Xiu Dai,,$^{a}$ Jun Gu, $t^{a}$ Li-Qun Kang, ${ }^{a}$ Yu-Hao Wang, ${ }^{a}$ Rui Si, iD ${ }^{b}$ \\ Ze-Qiong Zhao, ${ }^{a}$ Wen-Chi Liu, ${ }^{a}$ Xuefeng Fu, ${ }^{a}$ Ling-Dong Sun, ${ }^{\text {a }}$ Ya-Wen Zhang ${ }^{\text {*a }}$ \\ and Chun-Hua Yan (D)*a
}

\begin{abstract}
For converting a homogeneous synthetic chemistry reaction to a heterogeneous one, using nanocatalysts, it is essential to investigate the underlying principles and the associated dominant factors of methodologies between inorganic nanoparticles and organic substrates. Here it is reported that novel, hydrothermally synthesized ruthenium (Ru) nanoparticles performed differently in molecular oxygen activation via single electron transfer for cross-dehydrogenative-coupling reactions, following a volcano shaped relationship between their oxidation levels and catalytic activity. Characterizations and a systematic mechanism study indicated that $\mathrm{Ru}^{3+}$ sites contributed to more superoxo- or peroxo-like species being formed from molecular oxygen on Ru nanoparticles with a moderate oxidation level, and the $\mathrm{Ru}^{3+}$ were the highly active species which accelerate this cross-dehydrogenative-coupling reaction via single electron transfer.
\end{abstract}

Received 21st May 2017

Accepted 13th June 2017

DOI: 10.1039/c7ra05726a

rsc.li/rsc-advances significance to develop highly efficient nanocatalysts for important organic reactions.

Carbon-carbon bond formation is the essential link in all organic molecules, and is a central part of many chemical syntheses, such as in natural products, medicines, and organic materials. ${ }^{15-20}$ Building a $\mathrm{C}-\mathrm{C}$ linkage directly via the cleavage of dual $\mathrm{C}-\mathrm{H}$ bonds using molecular oxygen as the terminal oxidant, called aerobic cross-dehydrogenative coupling (CDC), has been successfully developed into the most atom-economic, clean, and efficient strategy in synthetic organic chemistry. ${ }^{15,21,22}$ Homogeneous transition metal catalyzed activation of $\mathrm{C}-\mathrm{H}$ bonds for $\mathrm{C}-\mathrm{C}$ bond formation, has proved to be versatile for the CDC reaction, using metals such as cobalt (Co), copper ( $\mathrm{Cu})$, iron (Fe), nickel (Ni), palladium $(\mathrm{Pd})$, rhodium $(\mathrm{Rh})$, and ruthenium $(\mathrm{Ru}) .{ }^{23-25}$ However, for widely investigated $\mathrm{Pd}$ or $\mathrm{Cu}$ catalyzed $\mathrm{CDC}$ reactions, most of the reports only provided possible reaction mechanisms without enough experimental evidence. In past decades, the development of heterocatalysis means that nanoparticles (NPs) play more and more important roles in organic syntheses, and many NPs (e.g., $\mathrm{Fe}_{3} \mathrm{O}_{4}, \mathrm{Cu}_{6} \mathrm{Se}_{4.5}, \mathrm{CuFe}_{2} \mathrm{O}_{4}$, ruthenium(Iv) oxide $\left(\mathrm{RuO}_{2}\right), \mathrm{RuO}_{2}$ /graphene, and so) exhibit higher catalytic performance than their salts or complexes in CDC reactions. ${ }^{26-29}$ However, the complicated structure of the active sites makes the elucidation of the reaction mechanism in a heterogeneous system challenging. ${ }^{30-32}$ Thus, understanding the relationship between the structure effects (electronic and geometric) of NPs and the catalytic performance towards important organic reactions is necessary to illustrate the

\footnotetext{
${ }^{a}$ Beijing National Laboratory for Molecular Sciences, State Key Laboratory of Rare Earth Materials Chemistry and Applications, PKU-HKU Joint Laboratory in Rare Earth Materials and Bioinorganic Chemistry, College of Chemistry and Molecular Engineering, Peking University, Beijing 100871, China. E-mail: yan@pku.edu.cn; ywzhang@pku.edu.cn; sun@pku.edu.cn

${ }^{b}$ Shanghai Synchrotron Radiation Facility, Shanghai Institute of Applied Physics, Chinese Academy of Sciences, Shanghai 201204, China

$\dagger$ Electronic supplementary information (ESI) available. See DOI: 10.1039/c7ra05726a

\$ These authors contributed to this work equally.
} 
reaction mechanism and to enable the screening of highly efficient heterocatalysts.

In this paper it is reported that novel $\mathrm{Ru}$ NPs performed differently in triggering CDC reactions. The catalytic mechanism was studied and it was found that the content of $\mathrm{Ru}^{3+}$ and its oxidation levels were crucial for the catalytic activity, and a volcano shaped relationship was established. On the basis of systematic characterizations, adsorbed superoxo- or peroxo-like species depending on the content of $\mathrm{Ru}^{3+}$ drove the CDC reactions, which was the essence of the as-observed structureactivity relationship.

\section{Results and discussion}

Ru NPs used in this work were prepared using a hydrothermal strategy, in which ruthenium chloride $\left(\mathrm{RuCl}_{3} \cdot x \mathrm{H}_{2} \mathrm{O}\right)$, formaldehyde and poly(vinylpyrrolidone) (PVP) were used as precursor, reducing agent and particle stabilizer, respectively. Sodium oxalate $\left(\mathrm{Na}_{2} \mathrm{C}_{2} \mathrm{O}_{4}\right)$, which can form stable complexes with $\mathrm{Ru}^{2+}$ and $\mathrm{Ru}^{3+}$ ions, ${ }^{33}$ was also introduced to avoid the rapid and complete reduction of the $\mathrm{Ru}$ precursor. Therefore, it was expected that $\mathrm{Ru}$ NPs with a considerable amount of $\mathrm{Ru}$ atoms with a positive valency would be obtained. The Ru NPs obtained after $8 \mathrm{~h}$ (denoted as Ru-NP-1) mainly contains $70 \mathrm{wt} \%$ of hexagonal-closest-packed (hcp) ultra-thin (thickness of $1.4 \mathrm{~nm}$ ) plates with two $\{0001\}$ facets as a basal surface, and the other $30 \mathrm{wt} \%$ of the NPs was comprised of poorly crystallized nanospheres. Transmission electron spectroscopy (TEM) images, and X-ray diffraction (XRD) patterns were presented in the ESI; Fig. S1 and S2, $\uparrow$ and the size distributions of all the samples are listed in Table S1. $\dagger$

The $\mathrm{Ru}$ NPs were introduced into the CDC reaction of tetrahydroisoquinolines via oxidative $\mathrm{C}\left(\mathrm{sp}^{3}\right)-\mathrm{H}$ functionalization. ${ }^{34-36}$ With the optimization of reaction conditions (Table S5; ESI $\dagger$ ), the Ru-nanocatalyzed CDC reaction of tetrahydroisoquinolines and nucleophiles such as indole and nitromethane performed with excellent selectivity and reactivity (Fig. 1). These Ru NPs converted the traditionally homogeneous CDC reaction with tough oxidants, energy supplement or/and high temperature into a heterogeneous process with a simple procedure, namely, just stirring under ambient conditions (room temperature, cheap solvents methanol $\left(\mathrm{CH}_{3} \mathrm{OH}\right)$ /water $\left(\mathrm{H}_{2} \mathrm{O}\right)$, open flask, and free of light or thermal energy supplement). Turnover frequency (TOF) was as high as 33.6 $\mathrm{s}^{-1}$ under $60{ }^{\circ} \mathrm{C}$. The recycling capability of $\mathrm{Ru}$ NPs was also examined, and the yields of recovered Ru NPs changed slightly. The listed yields of product 4 a were $97 \%$, 93\%, 87\%, 96\%, 86\%, $77 \%$ after six catalytic cycles.

It is difficult to distinguish homogeneous and heterogeneous catalysis, because of the possibility that metal ions may leach from a heterogeneous catalyst into solution and act as the homogeneous active species. Therefore, it was necessary to test the $\mathrm{Ru}$ ions leached from NPs in the reaction system. Firstly, as revealed by using in situ X-ray adsorption spectroscopy (see Fig. 2), the spectra of the sample at different reaction times did not show obvious differences, indicating that the oxidation level of the Ru element in the NPs remained unchanged during the catalytic reaction. Secondly, no Ru element was detected using inductively coupled plasma - atomic emission spectroscopy
(1)
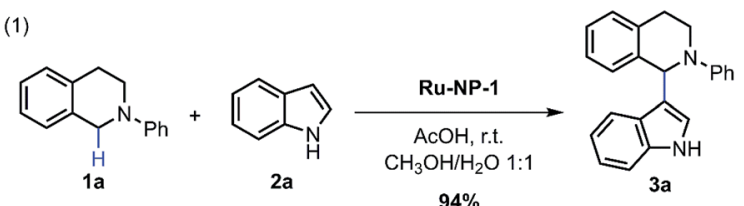

$2 a$

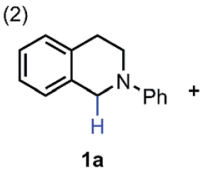

$\mathrm{CH}_{3} \mathrm{NO}_{2}$

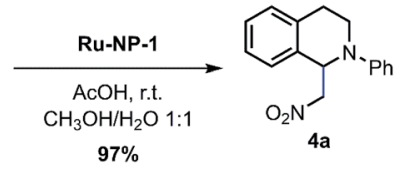

Fig. 1 Ru-nanocatalyzed CDC reactions. Reaction conditions: 8 mol\% of Ru NPs, 1 equiv. of 1, 4 equiv. of 2, $48 \mu \mathrm{L}$ acetic acid (AcOH), $0.025 \mathrm{M}$ in concentration. Isolated yield of $3 a$ after column chromatography and the yield of product $4 \mathrm{a}$ was determined using ${ }^{1} \mathrm{H}-\mathrm{NMR}$ with 4 chloroacetophenone as an internal standard.

(ICP-AES) in the reaction solvent mixture of $\mathrm{CH}_{3} \mathrm{OH}-\mathrm{H}_{2} \mathrm{O}$ after removal of $\mathrm{Ru}$ NPs. Thirdly, no significant changes in the morphology and oxidation level were observed, as revealed by the TEM image and X-ray photoelectron spectroscopy (XPS) spectra shown in Fig. S3 and S4 (ESI†), indicating that Ru NPs were stable during this reaction. Finally, the recovered solution was also used after removing Ru NPs to catalyse the model substrates and no reaction occurred. Thus, it was verified that the $\mathrm{Ru}$ NPs did not leach to form a homogeneously active catalyst.

A study of the mechanism of the heterogeneous CDC reaction helped to understand the catalytic behaviour and function of NPs towards organic substrates. The results of control experiments used to test the importance of oxygen are presented in Table S7. $\dagger$ The results confirmed that oxygen is necessary in this reaction and more oxygen yields a faster conversion rate of substrates. Furthermore, electron paramagnetic resonance (EPR) spectroscopy showed the existence of superoxide radicals $\left(\mathrm{O}_{2}{ }^{-}\right)$that were generated when oxygen molecules captured one electron in this reactive system, but no

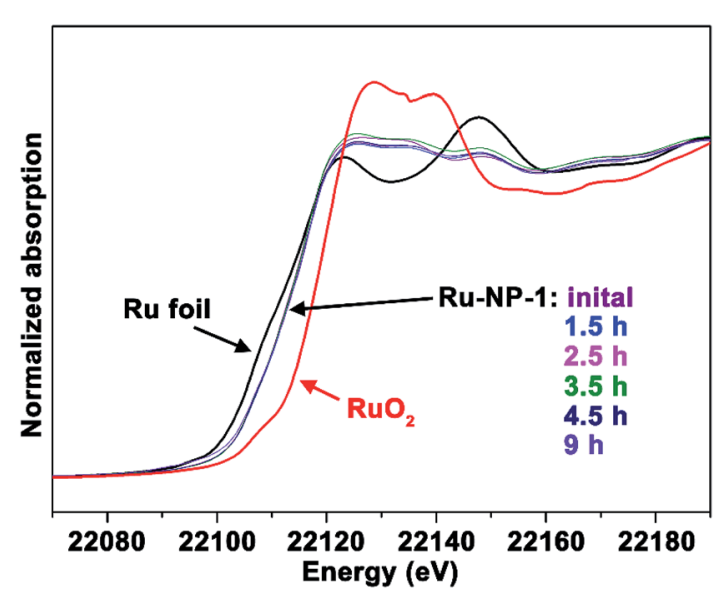

Fig. 2 Ru K-edge in situ X-ray adsorption spectroscopy monitoring of Ru-NP-1 during the catalytic reaction. The spectra were taken before the reaction and during the reaction at of $1.5 \mathrm{~h}, 2.5 \mathrm{~h}, 3.5 \mathrm{~h}, 4.5 \mathrm{~h}$ and $9 \mathrm{~h}$. The spectra of the $\mathrm{Ru}$ foil and $\mathrm{RuO}_{2}$ powders were used as references. 


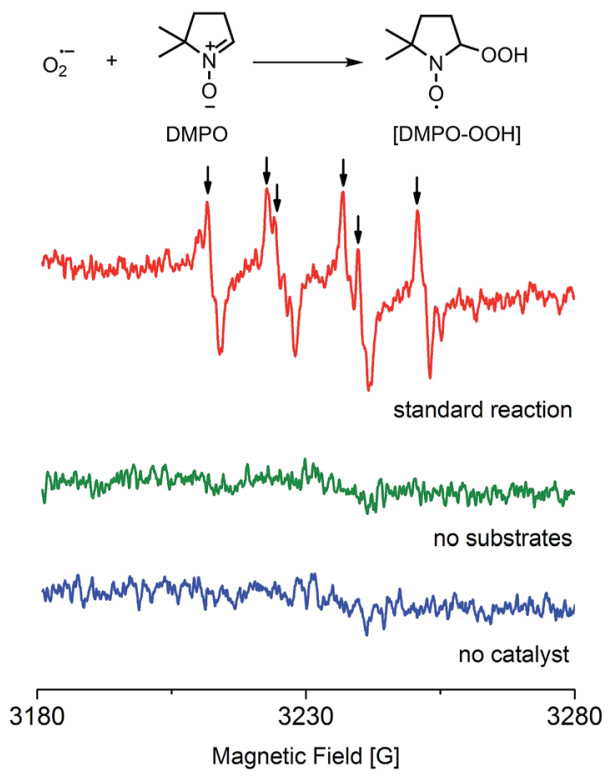

Fig. 3 EPR spectrum of [DMPO-OOH] formed in the standard reactive system of Ru-nanocatalyzed CDC reactions (microwave frequency: $9056.103 \mathrm{MHz}$, microwave power: $4.0 \mathrm{~mW}$ ). There are classical six peaks of the signals corresponding to [DMPO-OOH]. The calculated hyperfine splittings are $g_{0}$ (2.002), $\alpha_{H}(12.5 \mathrm{G}), \alpha_{N}(14.0 \mathrm{G})$.

radicals were detected when the substrates or the catalyst was absent (Fig. 3)..$^{37}$ Thus, $\mathrm{O}_{2}+\mathrm{e}^{-}=\mathrm{O}_{2}{ }^{\cdot-}$, and this process not only suggests that this CDC reaction proceeds as a single-electrontransfer (SET) catalysis, but also confirmed that oxygen was the electron acceptor.

Some previous work has shown that some adsorbed radicals, especially reactive oxygen species, can form on Ru-based heteroor homogeneous catalysts and are active sites for the catalytic reaction. ${ }^{38,39}$ In order to study the relationship between the

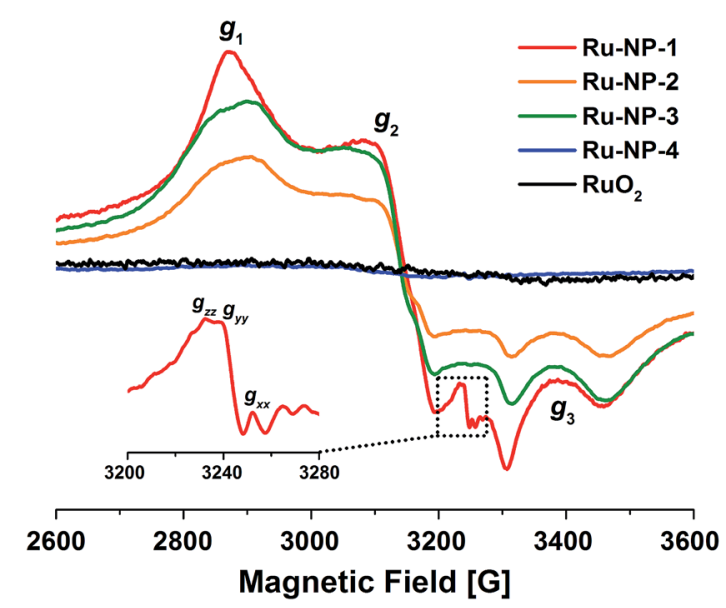

Fig. 4 EPR spectra of Ru-NP-1 (red curve), Ru-NP-2 (orange curve), Ru-NP-3 (green curve) and Ru-NP-4 (blue curve) all as solid powders and $\mathrm{RuO}_{2}$ powders (black curve) of the same mass collected at $123 \mathrm{~K}$. For Ru-NP-1, a signal was superimposed on the signal of $\mathrm{Ru}^{3+}$ species $\left(g_{1}=2.23, g_{2}=2.04, g_{3}=1.86\right)$, which was ascribed to the superoxoor peroxo-like species $\left({ }^{*} \mathrm{O}_{2}{ }^{-}\right)$adsorbed on the Ru catalyst, as shown in the inset. structure and the activity of the Ru NPs in the solution, EPR was used to detect radicals on the surface of Ru NPs which possibly mediated the SET process (Fig. 4). First, very broad and intensive signals with $g$ tensor components $g_{1}=2.19, g_{2}=2.04$ and $g_{3}$ $=1.88$ ascribed to the $\mathrm{Ru}^{3+}$ species were observed on Ru-NP-1. If the as-synthesized Ru-NP-1 was post-reduced using hydrogen $\left(\mathrm{H}_{2}\right)$ under hydrothermal conditions (denoted as Ru-NP-2), the signal of $\mathrm{Ru}^{3+}$ became weaker than that of Ru-NP-1 with the same amount of Ru element. The $\mathrm{RuO}_{2}$ powders considered as the over-oxidized sample showed little sign of $\mathrm{Ru}^{3+}$ species. Meanwhile, the SET reactions were used to test the catalytic activity of the three Ru catalysts. Among the Ru nanocatalysts, Ru-NP-1 showed the highest TOF value (up to $33.6 \mathrm{~s}^{-1}$ ), and the reaction achieved the final product with full conversion and an excellent yield as high as $94 \%$ (Table 1). Thus, it was supposed that the content of $\mathrm{Ru}^{3+}$ in the $\mathrm{Ru}$ catalysts was related to the catalytic activity.

$\mathrm{Ru}$ NPs with different $\mathrm{Ru}^{3+}$ contents were synthesized by just prolonging the time of synthesis of Ru-NP-1 to $16 \mathrm{~h}$ and $24 \mathrm{~h}$, denoted as Ru-NP-3 and Ru-NP-4, respectively. As shown in Fig. S1 (ESI) and Table S1 (ESI $\dagger$ ), the thickness of the plateshaped NPs in these two samples remained the same as that of Ru-NP-1 (1.4 nm), and the morphology selectivity of the plates were also similar ( $77 \mathrm{wt} \%$ for Ru-NP-3 and $78 \mathrm{wt} \%$ for Ru-NP-4). The EPR results showed that the $\mathrm{Ru}^{3+}$ content of Ru catalysts with the same mass was Ru-NP-1 > Ru-NP-3 > Ru-NP-2 > Ru-NP-4 $>\mathrm{RuO}_{2}$ (see the magnified detail in Fig. 4). Table 1 compares the apparent TOF of different catalysts, which is defined as the average number of reactant molecules consumed on one surface Ru site per second (see ESI $\dagger$ for the calculation process). Different $\mathrm{Ru}$ nanocatalysts exhibited quite different catalytic activities under the same ambient conditions. The higher the content of $\mathrm{Ru}^{3+}$ in the nanocatalysts is, the higher the catalytic activity toward CDC reactions is.

More importantly, an obvious signal ascribed to adsorbed superoxo- or peroxo-like species $\left({ }^{*} \mathrm{O}_{2}{ }^{\cdot-}\right)^{40-42}$ was observed in the EPR spectrum of Ru-NP-1, the sample with highest content of $\mathrm{Ru}^{3+}$ species. The ${ }^{*} \mathrm{O}_{2}{ }^{--}$species might form because of the electron transfer from the Ru sites on the surface of Ru NPs to oxygen molecules, as shown by:

$$
\left(\mathrm{Ru}^{n+}\right)_{\text {surf }}+\mathrm{O}_{2} \rightarrow\left(\mathrm{Ru}^{(n+1)+}\right)_{\text {surf }}-{ }^{*} \mathrm{O}_{2} \cdot-
$$

This process is more likely to occur on $\mathrm{Ru}^{3+}$ sites on the surface of Ru NPs. The signals of the ${ }^{*} \mathrm{O}_{2}{ }^{-{ }^{-}}$species were not detected for Ru-NP-2 and $\mathrm{RuO}_{2}$, which had a lower $\mathrm{Ru}^{3+}$ content. If benzoquinone was added to the reaction as a radical quencher of ${ }^{*} \mathrm{O}_{2}{ }^{-}$species, no reactions occurred on Ru-NP-1 (entry 4 in Table S7, ESI $\dagger$ ), indicating that the ${ }^{*} \mathrm{O}_{2}{ }^{-{ }^{-}}$species mediated the SET process. Based on the previous characterizations, it is proposed that the oxidation level of Ru nanocatalysts determines the content of $\mathrm{Ru}^{3+}$ sites on which ${ }^{*} \mathrm{O}_{2}{ }^{--}$species can form and mediate the SET process. Therefore, Ru nanocatalysts with a moderate oxidation level may trigger the CDC reactions of tetrahydroisoquinolines and achieve excellent catalytic performances. 
Table 1 Catalytic properties of the cross-dehydrogenative-coupling reactions with different Ru catalysts ${ }^{a}$

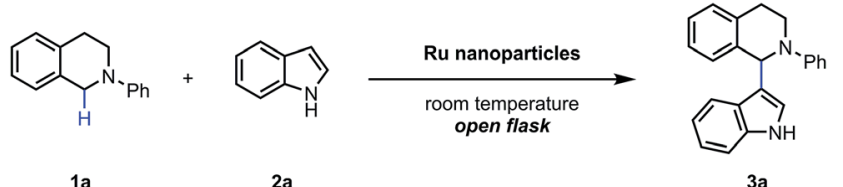

$1 \mathrm{a}$

2a

3a

\begin{tabular}{|c|c|c|c|c|c|c|}
\hline Entry & Catalyst & Equivalent $^{b}[\mathrm{~mol} \%]$ & Time $[\mathrm{h}]$ & Conversion [\%] & Yield $^{c}[\%]$ & $\mathrm{TOF}^{d}\left[\mathrm{~s}^{-1}\right]$ \\
\hline 1 & Ru-NP-1 & 8 & 42 & 100 & 94 & 1.6 \\
\hline 2 & Ru-NP-1 & 8 & 2 & 100 & 93 & $33.6^{e}$ \\
\hline 3 & Ru-NP-2 & 8 & 72 & 41 & 34 & 0.28 \\
\hline 4 & Ru-NP-3 & 8 & 72 & 73 & 57 & 0.45 \\
\hline 5 & $\mathrm{Ru}-\mathrm{NP}-4$ & 8 & 72 & 74 & 45 & 0.10 \\
\hline 6 & $\mathrm{RuO}_{2}$ & 80 & 45 & 30 & 18 & 0.091 \\
\hline
\end{tabular}

${ }^{a}$ Reaction conditions: $0.10 \mathrm{mmol}$ of $1 \mathrm{a}, 4$ equiv. of $2 \mathrm{a}, 48 \mu \mathrm{L}$ of $0.025 \mathrm{M} \mathrm{AcOH}$, solvent: $\mathrm{H}_{2} \mathrm{O} / \mathrm{CH}_{3} \mathrm{OH}(1: 1)$, room temperature. ${ }^{b}$ Molar ratio of Ru element to reagent 1a. ICP-AES was used for the quantification of the Ru element. ${ }^{c}$ Isolated yield after column chromatography. ${ }^{d}$ The TOF was obtained with the conversion of substrate 1a being lower than $15 \%$. The methods to calculate the number of catalytic sites are shown in the ESI. ${ }^{e}$ Reaction temperature was $60^{\circ} \mathrm{C}$.

The content of $\mathrm{Ru}^{3+}$ depends on the oxidation level of $\mathrm{Ru}$ NPs. To investigate the relationship between the catalytic activity and oxidation levels of Ru NPs, an X-ray adsorption fine structure (XAFS) analysis of different $\mathrm{Ru}$ nanocatalysts was performed. Fig. 5a shows the X-ray absorption near edge structure (XANES) spectra of Ru-NP-1, Ru-NP-2, Ru-NP-3, Ru-NP4, $\mathrm{Ru}$ foil and $\mathrm{RuO}_{2}$. The higher energy of the $\mathrm{Ru} \mathrm{K}$-edge reflects its higher oxidation level. Thus, the oxidation levels of different $\mathrm{Ru}$ NPs were in the sequence of Ru-NP-3 > Ru-NP-1 > Ru-NP-2 > Ru-NP-4. This result was consistent with the $R$-space extended X-ray absorption fine structure (EXAFS) data shown in Fig. 5b. Fourier transformation (F.T.) of the $k_{2}$-weighted extended EXAFS data showed two main peaks around $2.0 \AA$ and $2.7 \AA$, attributed to $\mathrm{Ru}-\mathrm{O}$ and $\mathrm{Ru}-\mathrm{Ru}$ scattering, respectively. ${ }^{43}$ The ratio of the peak intensity of $\mathrm{Ru}-\mathrm{O}$ scattering to $\mathrm{Ru}-\mathrm{Ru}$

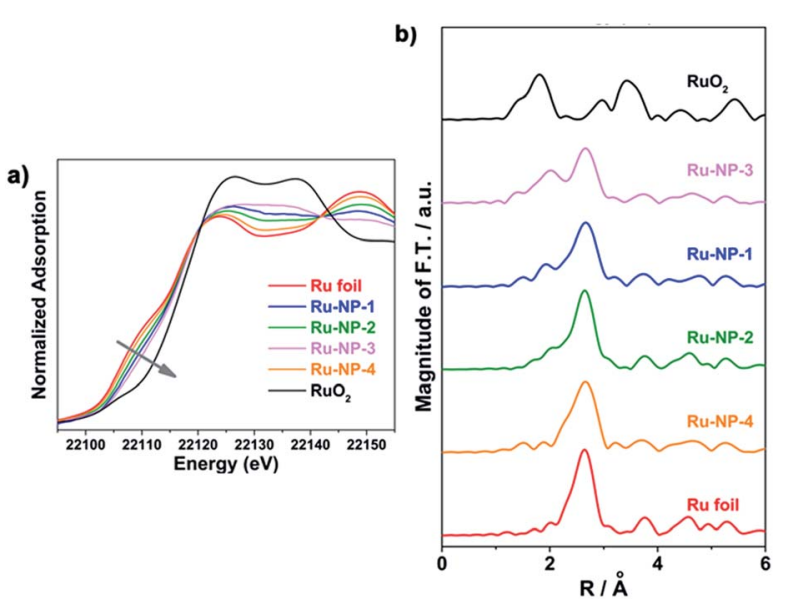

Fig. 5 (a) XANES spectra and (b) $R$-space EXAFS spectra and the curve fitting for Ru K-edge of Ru foil (red curve), Ru-NP-1 (blue curve), RuNP-2 (green curve), Ru-NP-3 (pink curve), Ru-NP-4 (orange curve) and $\mathrm{RuO}_{2}$ (black curve). scattering reflected the sequence of oxidation levels, which showed the same trend as XANES. Combined with the activity data of the SET reactions, Ru-NP-1 with a moderate oxidation level showed the highest catalytic activity.
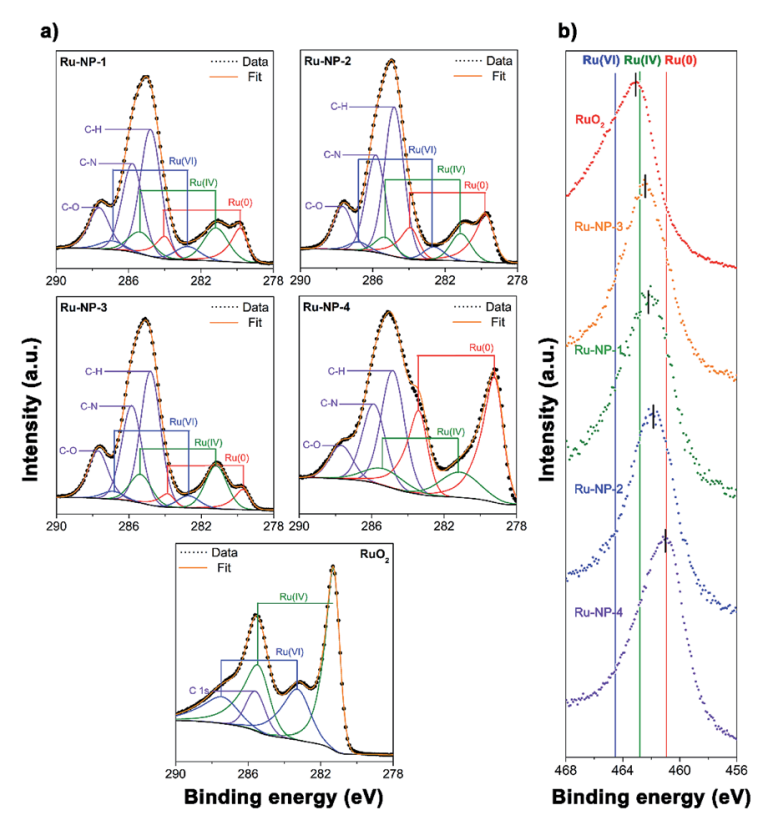

Fig. 6 XPS spectra of Ru-NP-1, Ru-NP-2, Ru-NP-3, Ru-NP-4 and $\mathrm{RuO}_{2}$ powders. (a) The spectra in the $\mathrm{C}-1 \mathrm{~s}$ and $\mathrm{Ru}-3 \mathrm{~d}$ regions. Each spectrum was deconvoluted into $\mathrm{C}$-1s peaks at $284.8 \mathrm{eV}, 285.8 \mathrm{eV}$ and $287.6 \mathrm{eV}$ (violet curves), which were assigned to $\mathrm{C}$ atoms in the $\mathrm{C}-\mathrm{H}, \mathrm{C}-\mathrm{N}$ and $\mathrm{C}=\mathrm{O}$ forms, respectively, and Ru-3d doublets $\left(3 d_{5 / 2}\right.$ and $3 d_{3 / 2}$, $\Delta=4.17 \mathrm{eV}$ ) with the binding energy of Ru $3 \mathrm{~d}_{5 / 2}$ peak at $279.5-279.9 \mathrm{eV}$, 281.2-281.4 eV and 282.6-282.8 eV which were assigned to Ru(0) (red curves), Ru(Iv) (green curves) and Ru(vi) (blue curves) states, respectively. ${ }^{45-47}$ The fitting results of the Ru-3d spectra are shown in Table 2. (b) The spectra in the Ru-3p $p_{3 / 2}$ region. The red, green and blue vertical lines indicate the binding energy of $\mathrm{Ru}(0), \mathrm{Ru}(\mathrm{Iv})$ and $\mathrm{Ru}(\mathrm{vI})$ states, respectively. ${ }^{48,49}$ 
Table 2 XPS data fitting results of all the catalysts in $\mathrm{Ru}-3 \mathrm{~d}$ region

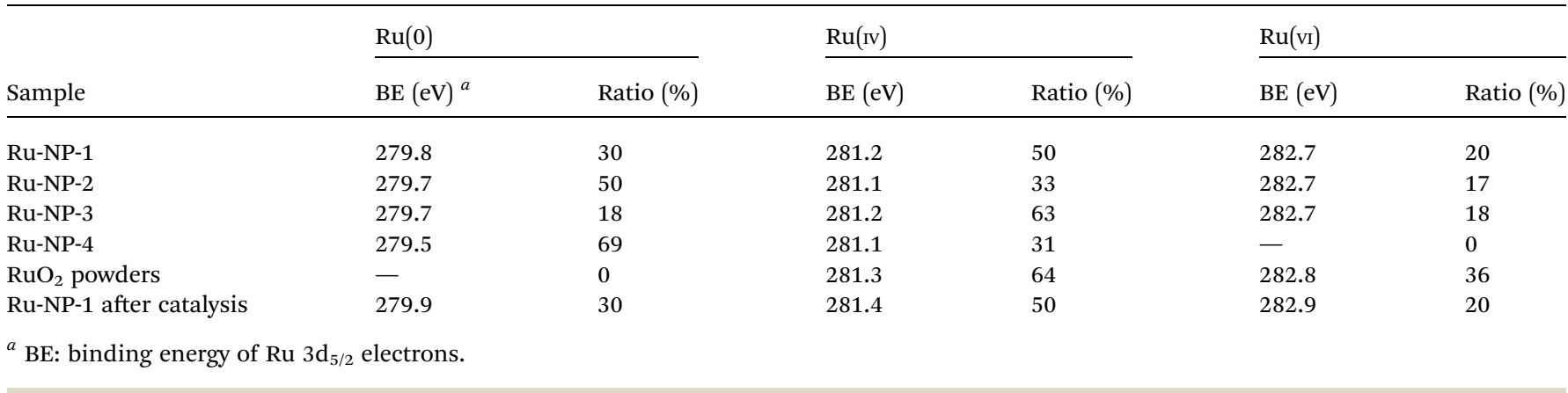

Next, XPS was used to investigate the surface oxidation level of the Ru NPs. Considering the probing depth of XPS and the ultra-thin nature of the Ru NPs, most Ru atoms in the sample could be detected in the analysis. As shown in Fig. 6a, the C-1s and $\mathrm{Ru}-3 \mathrm{~d}$ regions of the samples were deconvoluted into three $\mathrm{C}-1 \mathrm{~s}$ peaks at $284.80 \mathrm{eV}(\mathrm{C}-\mathrm{H}), 285.80 \mathrm{eV}(\mathrm{C}-\mathrm{N})$ and $287.63 \mathrm{eV}$ $(\mathrm{C}=\mathrm{O})$, and three $\mathrm{Ru}-3 \mathrm{~d}$ doublets $\left(3 \mathrm{~d}_{5 / 2}\right.$ and $\left.3 \mathrm{~d}_{3 / 2}, \Delta=4.17 \mathrm{eV}\right)$ with the binding energy (BE) of $\mathrm{Ru} 3 \mathrm{~d}_{5 / 2}$ peak at $279.5-279.9 \mathrm{eV}$, 281.2-281.4 $\mathrm{eV}$ and 282.6-282.8 eV, assigned to the $\mathrm{Ru}(0), \mathrm{Ru}(\mathrm{IV})$ and $\mathrm{Ru}(\mathrm{vI})$ states, respectively. The fitting results of the XPS spectra of Ru-3d electrons are listed in Table 2. Compared with Ru-NP-1, the post-reduced sample (Ru-NP-2) showed a significantly lower oxidation level. The oxidation level of Ru-NP-3 was higher than that of Ru-NP-1, probably because of the oxidizing effect of the oxygen sealed in the reactor, on the Ru NPs from $8 \mathrm{~h}$ to $16 \mathrm{~h}$. As the reaction time was extended to $24 \mathrm{~h}$, the Ru-NP-4 obtained showed greatly enhanced crystallinity (Fig. S2, ESI $\dagger$ ) and became inert and were unable to be oxidized. ${ }^{44}$ Thus, $\mathrm{Ru}-$ NP-4 represented the lowest oxidation level. The XPS spectra in the $\mathrm{Ru}-3 \mathrm{p}$ regions showed the same trend of the oxidation levels of different Ru catalysts, as shown in Fig. $6 \mathrm{~b}$.

When the TOF values of all the Ru catalysts were plotted against their oxidation level (quantified as the percentage of surface Ru atoms with positive valences among all the surface $\mathrm{Ru}$ atoms deduced from the Ru-3d XPS spectra), a volcano shaped relationship was found, as shown in Fig. 7. The highest catalytic activity was achieved at a moderate oxidation level. The catalytic performances and the oxidation level of Ru NPs with other morphologies synthesized by hydrothermal methods were also investigated, and the results obtained agreed well with the volcano shaped relationship, as discussed in Fig. S5 and S6 (ESI) and Tables S2 and S6 (ESI $\dagger$ ).

In this work, $\mathrm{Ru}$ nanocatalysts were synthesized using a hydrothermal method. With the understanding of the growth mechanism of the Ru NPS ${ }^{33}$ (Fig. S11-S13, ESI $\dagger$ ), after the nucleation and growth steps, Ru NPs were oxidized mainly using subcritical water in hydrothermal synthesis, ${ }^{50-53}$ together with the generation of active ${ }^{*} \mathrm{O}_{2}{ }^{\cdot-}$ species at the surfaces of the $\mathrm{Ru} \mathrm{NPs}$ as revealed by the EPR measurements. $\mathrm{Ru}(0)$ was still the body constituent to support the structure and morphology of these Ru nanocatalysts with different oxidation levels. In the interface, the oxidized $\mathrm{Ru}$ nanocatalysts triggered the CDC reaction of tetrahydroisoquinoline derivatives via the SET process. The volcano shaped relationship between the catalytic activity and the oxidation level confirmed the as-proposed process on how Ru NPs catalyze the CDC reactions: the ${ }^{*} \mathrm{O}_{2}{ }^{\cdot-}$ species formed on $\mathrm{Ru}^{3+}$ sites on the surface of Ru NPs were the active sites to mediate the SET process and connected the inorganic nanocatalysts and organic substrates. For the completely reduced Ru NPs, a few $\mathrm{Ru}^{3+}$ sites existed on the

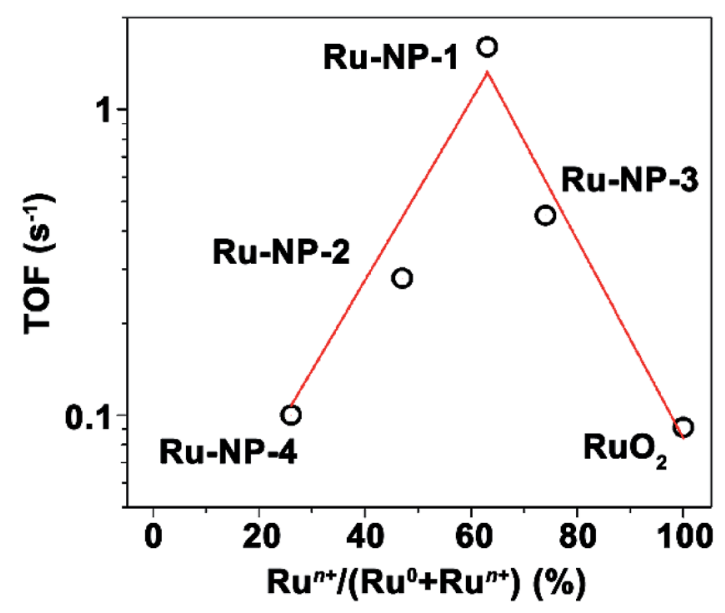

Fig. 7 Plot of the TOF values of all the catalysts against their oxidation level quantified as the percentage of surface Ru atoms with positive valencies among all the surface Ru atoms deduced from the Ru-3d XPS spectra. A volcano shaped relationship between the TOF values and the oxidation level is indicated by the red line.

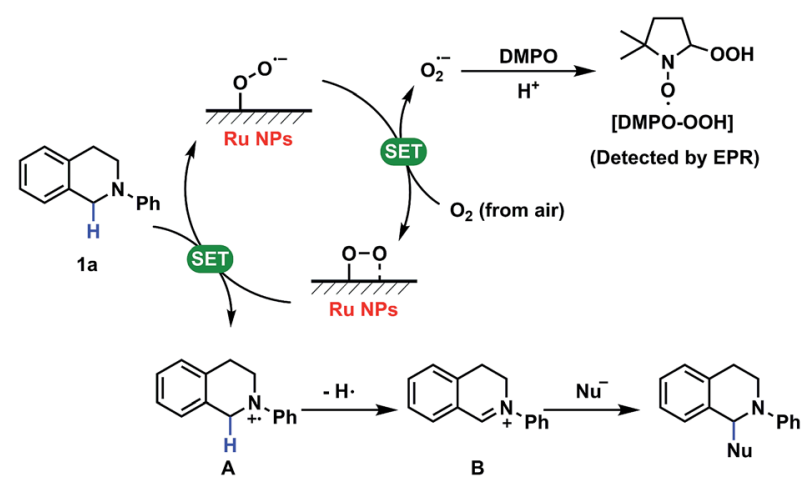

Scheme 1 Mechanism of SET reactions catalyzed by Ru NPs. 
surface, whereas the $\mathrm{Ru}^{3+}$ sites first increased with increasing degree of oxidation of Ru NPs, and then decreased as the $\mathrm{Ru}^{4+}$ sites became dominant on the surface. The content of $\mathrm{Ru}^{3+}$ sites was highest on Ru NPs with a moderate oxidation level, which consequently generated more ${ }^{*} \mathrm{O}_{2}{ }^{\cdot-}$ species by the electron transfer from oxygen molecules to the $\mathrm{Ru}^{3+}$ sites and the highest catalytic activity for the CDC reaction was exhibited with the sample of Ru-NP-1.

Based on the previous discussion, it is now possible to systematically clarify the mechanism as shown in Scheme 1. In the SET process, Ru NPs with superoxo- or peroxo-like species captured one single electron from tetrahydroisoquinoline derivative 1a with lone pair electrons to generate aminium radical cation $\mathrm{A}$ and transferred it to oxygen from the air to form the superoxide anion radical $\left(\mathrm{O}_{2}{ }^{-}\right)$, which is the impetus of this reaction. Then radical intermediate $\mathrm{B}$ could be achieved by several conversions following the formation of aminium radical ion A and attack by nucleophiles yielding the final complex molecules. In this heterogeneous catalysis, it is supposed that a large amount of reactive oxygen radicals on the surface of $\mathrm{Ru}$ NPs, that are associated with their oxidation level, could drive the catalytic

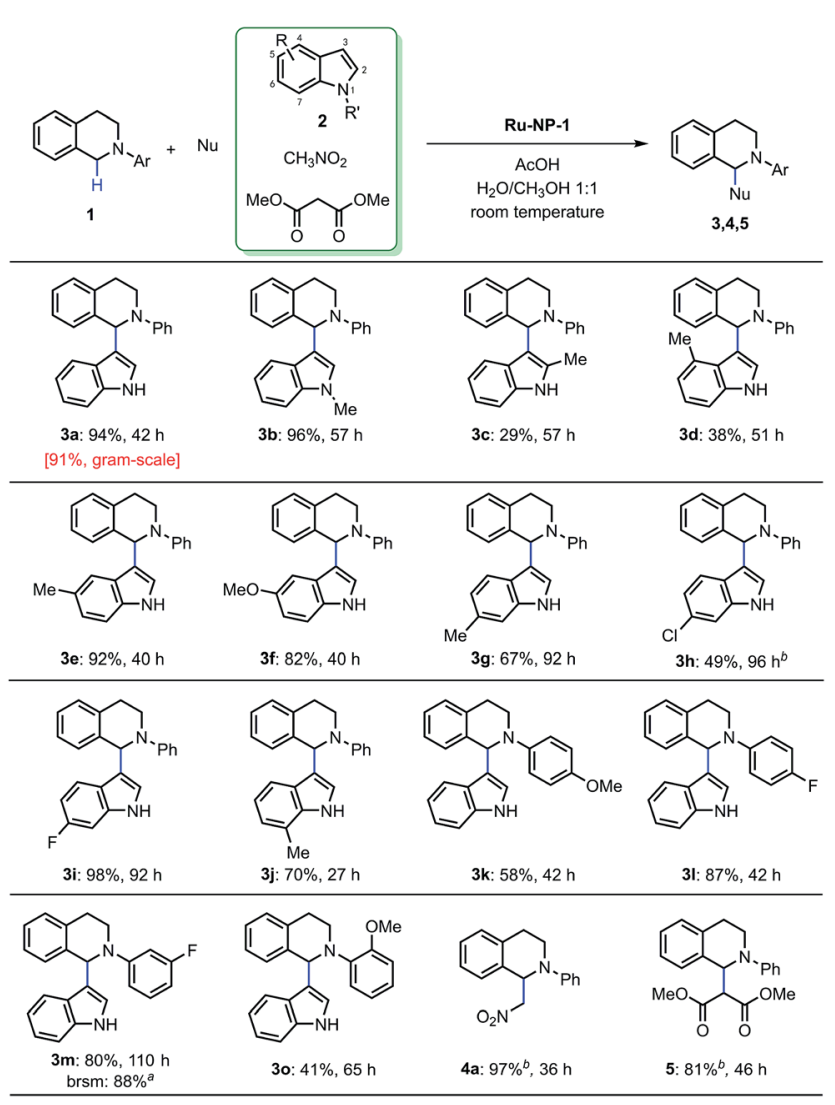

Fig. 8 Ru NP catalyzed CDC reactions. Reaction conditions: 8 mol\% of $\mathrm{Ru}$ nanocatalyst, 1 equiv. of 1,4 equiv. of $2,10-48 \mu \mathrm{L}$ of $\mathrm{AcOH}$, $0.025 \mathrm{M}$ in concentration. Isolated yields were obtained after column chromatography, except that the yields of products $4 a$ and 5 were determined using ${ }^{1} \mathrm{H}$-NMR using 4-chloroacetophenone as an internal standard. ${ }^{a}$ Recovered $10 \%$ of $1 \mathrm{~d}, 88 \%$ of yield based on the recovered starting material (brsm). ${ }^{b}$ Solvent of $3 \mathrm{~h}, 4 \mathrm{a}$ and 5 is $\mathrm{CH}_{3} \mathrm{OH}$. cycle and enhance the activities of Ru NPs for the CDC reactions. The detailed explanations of the organic mechanism including control experiments and proposed pathways are shown in Table S7 (ESI) and Fig. S14 (ESI†).

The general mechanism of the Ru-nanocatalyzed CDC reactions was further explored using the standard conditions [8 mol\% Ru-NP-1, $0.10 \mathrm{mmol}$ of $\mathrm{N}$-phenyl tetrahydroisoquinoline derivatives, 4 equiv. of indoles, $10-48 \mu \mathrm{L}$ of $\mathrm{AcOH}, \mathrm{H}_{2} \mathrm{O} / \mathrm{CH}_{3} \mathrm{OH}(1: 1)$ as solvent, room temperature, concentration of $0.025 \mathrm{M}(\mathrm{ESI} \dagger)]$ in the optimization studies. Significantly, all the positions of the substituted nucleophilic indoles were reactive and the yields were generally high (Fig. 8 3b-3j). The product (3d) that substitutes the indole at the 4-position has not been discovered before. In addition, the substrates substituted $N$-phenyl tetrahydroisoquinolines at all positions of $N$-aryl group perform high reactivity and the obtained products (3k-3o) are also first discovered. The reaction proceeds satisfactorily resulting in the formation of the crossingcoupling products with excellent yields (4a and 5). All the experimental results mentioned previously, shows that Ru-NP-1 with a moderate oxidation level presented excellent catalytic performance and a broad applicability, by virtue of the powerful single electron grabbing capacity under ambient conditions.

\section{Conclusions}

A novel Ru nanocatalyst with a different oxidation level was synthesized using a hydrothermal strategy. The Ru NPs with a moderate oxidation level exhibited excellent reactivity and selectivity for the CDC reaction of tetrahydroisoquinolines under ambient conditions. This heterogeneous catalytic process was free of energy supplement, sacrificial reductants or sacrificial oxidants compared to homogeneous catalysis, and can be conducted at the gram scale. It was proposed and confirmed that there was a heterogeneous catalytic mechanism of Ru-nanocatalyzed CDC reactions. Ru NPs firstly capture one electron from amine substrates to generate an aminium radical cation and subsequently transfer the electron to the oxygen. Then, the active aminium radical cation could react with nucleophiles to form complex structures. The active aminium radical cation can be obtained for a series of available substrates. The catalytic behaviors present a volcano shaped correlation to the oxidation level of the NPs. The $\mathrm{Ru}^{3+}$ content was the highest for Ru NPs with a moderate oxidation level, which consequently generated more ${ }^{*} \mathrm{O}_{2}{ }^{-}{ }^{-}$species by electron transfer from $\mathrm{Ru}^{3+}$ sites to oxygen molecules and exhibited a higher catalytic activity to trigger this SET process.

By introducing catalytically active NPs into the SET process of CDC reactions, not only were the underlying principles and the associated dominant factors of nanocatalysis for the development of efficient and versatile approaches to heterogeneous catalysis under ambient conditions studied, but the results also provided a thorough insight into the relationship between the inorganic nanocatalysts and organic methodologies. Extended research with other NPs is necessary to facilitate the development of 
heterogeneous catalysts with high reactivity and selectivity in an even larger array of chemical reactions.

\section{Experiments}

\section{Hydrothermal synthesis of Ru nanocatalysts}

In a typical synthesis, $100 \mathrm{mg}$ of PVP and $80 \mathrm{mg}$ of $\mathrm{Na}_{2} \mathrm{C}_{2} \mathrm{O}_{4}$ were first dissolved in $10 \mathrm{~mL}$ of water, followed by addition $0.24 \mathrm{mmol} \mathrm{RuCl}{ }_{3} \cdot x \mathrm{H}_{2} \mathrm{O}, 0.062 \mathrm{~mL}$ of hydrochloric acid (1 M) and $0.1 \mathrm{~mL}$ of formaldehyde solution (40 wt\%). The solution was then diluted to $15 \mathrm{~mL}$, loaded in to a $25 \mathrm{~mL}$ Teflon-lined container and sealed in a matched steel autoclave. The autoclave was then heated in an oven kept at $160{ }^{\circ} \mathrm{C}$ for $8 \mathrm{~h}, 16 \mathrm{~h}$ or $24 \mathrm{~h}$ for the synthesis of Ru-NP-1, Ru-NP-3 and Ru-NP-4, respectively. After the reaction, the autoclave was cooled naturally to $25^{\circ} \mathrm{C}$ and $45 \mathrm{~mL}$ of acetone was added and the product was then collected by centrifugation at $7800 \mathrm{rpm}$ for $10 \mathrm{~min}$. The yield of Ru NPs using the hydrothermal method was measured using ICP-AES and found to be about $85 \%$.

A post-reduction process generating Ru-NP-2 was also used to lower the oxidation level of Ru-NP-1. The as-obtained Ru-NP-1 was dispersed in $15 \mathrm{~mL}$ of water containing $100 \mathrm{mg}$ of PVP. The dispersion was loaded into a $50 \mathrm{~mL}$ Teflon-lined reactor which was then filled with 2.0 MPa of $\mathrm{H}_{2}$. The reduction process was kept at $150{ }^{\circ} \mathrm{C}$ for $4 \mathrm{~h}$. After the reaction, $45 \mathrm{~mL}$ acetone was added and the NPs were collected by centrifugation at $7800 \mathrm{rpm}$ for $10 \mathrm{~min}$.

\section{General procedure of Ru-nanocatalyzed CDC reactions}

The concentration of a $\mathrm{Ru} \mathrm{NP}$ colloidal $\mathrm{CH}_{3} \mathrm{OH}$ solution was determined using ICP-AES. Ru nanocatalysts with the same Ru content ( $8 \mathrm{~mol} \%$ of the substrate content) were added into the solvents $\left[\mathrm{H}_{2} \mathrm{O} / \mathrm{CH}_{3} \mathrm{OH}(1: 1)\right]$ with $0.10 \mathrm{mmol}$ of tetrahydroisoquinoline derivatives, 4 equiv. of indoles and $10-48 \mu \mathrm{L}$ of AcOH. The mixture (concentration: $0.025 \mathrm{M}$ ) was stirred under room temperature. The reaction tube was immersed in water bath under room temperature. When the thin layer chromatography (TLC) indicated the disappearance of the starting material, the reaction mixture was filtered through a thin pad of silica gel. The filter cake was washed with petroleum ether/ethyl acetate, and the combined filtrate was concentrated. The crude product was purified using flash column chromatography on silica gel to give the final product.

\section{Acknowledgements}

The Authors wish to thank the National Natural Science Foundation of China (No. 21461162001, 21371011, 21621061, 21229101, 21573005 and 21331001), the National Basic Research Program of China (No. 2012CBA01204), the National Key Research and Development Program of China (no. 2016YFB0701100), the Beijing Natural Science Foundation (No. 2162019), and the 7th China Postdoctoral Science Foundation Funded Project (No. 2014T70009) for financial support. ML was also partly supported by a Postdoctoral Fellowship of PekingTsinghua Center for Life Sciences.

\section{Notes and references}

1 G. A. Somorjai, Chem. Rev., 1996, 96, 1223.

2 T. S. Ahmadi, Z. Wang, T. C. Green, A. Henglein and M. A. ElSayed, Science, 1996, 272, 1924.

3 Y. N. Xia, Y. J. Xiong, B. Lim and S. E. Skrabalak, Angew. Chem., Int. Ed., 2009, 48, 60.

4 C. Wang, H. Daimon, T. Onodera, T. Koda and S. H. Sun, Angew. Chem., Int. Ed., 2008, 47, 3588.

5 X. Wang, J. Zhuang, Q. Peng and Y. D. Li, Nature, 2005, 437, 121.

6 Y. Wu, D. S. Wang and Y. D. Li, Chem. Soc. Rev., 2014, 43, 2112.

7 X. Q. Huang, Z. P. Zhao, J. M. Fan, Y. M. Tan and N. F. Zheng, J. Am. Chem. Soc., 2011, 133, 4718.

8 A. R. Tao, S. Habas and P. D. Yang, Small, 2008, 4, 310.

9 K. M. Bratlie, H. Lee, K. Komvopoulos, P. D. Yang and G. A. Somorjai, Nano Lett., 2007, 7, 3097.

10 Y. Wu, S. F. Cai, D. S. Wang, W. He and Y. D. Li, J. Am. Chem. Soc., 2012, 134, 8975.

11 S. B. Wang, W. Zhu, J. Ke, M. Lin and Y. W. Zhang, ACS Catal., 2014, 4, 2298.

12 L. X. Dai, W. Zhu, M. Lin, Z. P. Zhang, J. Gu, Y. H. Wang and Y. W. Zhang, Inorg. Chem. Front., 2015, 2, 949.

13 M. Lin, L. Q. Kang, J. Gu, L. X. Dai, S. B. Tang, T. Zhang, Y. H. Wang, L. D. Li, X. Y. Zheng, W. Zhu, R. Si, X. F. Fu, L. D. Sun, Y. W. Zhang and C. H. Yan, Nano Res., 2017, 10, 922.

14 L. J. Durndell, C. M. A. Parlett, N. S. Hondow, M. A. Isaacs, K. Wilson and A. F. Lee, Sci. Rep., 2015, 5, 9425.

15 C. S. Yeung and V. M. Dong, Chem. Rev., 2011, 111, 1215.

16 J. M. Brown and N. A. Cooley, Chem. Rev., 1988, 88, 1031.

17 Y. N. Wu, J. Wang, F. Mao and F. Y. Kwong, Chem.-Asian J., 2014, 9, 26.

18 C. C. C. Johansson Seechurn, M. O. Kitching, T. J. Colacot and V. Snieckus, Angew. Chem., Int. Ed., 2012, 51, 5062.

19 S. A. Girard, T. Knauber and C. J. Li, From $C-H$ to $C-C$ Bonds: Cross-Dehydrogenative-Coupling, 2014.

20 X. Lu, B. Xiao, Z. Q. Zhang, T. J. Gong, W. Su, J. Yi, Y. Fu and L. Liu, Nat. Commun., 2016, 7, 11129.

21 C. Liu, H. Zhang, W. Shi and A. Lei, Chem. Rev., 2011, 111, 1780.

22 W. Shi, C. Liu and A. Lei, Chem. Soc. Rev., 2011, 40, 2761.

23 A. Meijere and F. Diederich, Metal-Catalyzed Cross-Coupling Reactions, Wiley-VCH, Weinheim, 2004.

24 C. A. Parrodi and P. J. Walsh, Angew. Chem., Int. Ed., 2009, 48, 467925.

25 Z. Shao and F. Peng, Angew. Chem., Int. Ed., 2010, 49, 9566. 26 S. Gupta, H. Joshi, N. Jain and A. K. Singh, J. Mol. Catal. A: Chem., 2016, 423, 135.

27 R. Hudson, S. Ishikawa, C. J. Li and A. Moores, Synlett, 2013, 24, 1637.

28 T. Zeng, G. Song, A. Moores and C. J. Li, Synlett, 2010, 13, 2002.

29 Q. Y. Meng, Q. Liu, J. J. Zhong, H. H. Zhang, Z. J. Li, B. Chen, C. H. Tung and L. Z. Wu, Org. Lett., 2012, 14, 5993. 
30 G. Ertl, Angew. Chem., Int. Ed. Engl., 1990, 29, 1219.

31 H.-J. Freund, G. Meijer, M. Scheffler, R. Schlögl and M. Wolf, Angew. Chem., Int. Ed., 2011, 50, 10064.

32 G. Ertl, Angew. Chem., Int. Ed., 2008, 47, 3524.

33 A. X. Yin, W. C. Liu, J. Ke, W. Zhu, J. Gu, Y.-W. Zhang and C.-H. Yan, J. Am. Chem. Soc., 2012, 134, 20479.

34 C.-J. Li, Acc. Chem. Res., 2009, 42, 335.

35 Q. Y. Meng, J.-J. Zhong, Q. Liu, X.-W. Gao, H.-H. Zhang, T. Lei, Z.-J. Li, K. Feng, B. Chen, C.-H. Tung and L.-Z. Wu, J. Am. Chem. Soc., 2013, 135, 19052.

36 K. Godula and D. Sames, Science, 2006, 312, 67.

37 J.-L. Clément, N. Ferré, D. Siri, H. Karoui, A. Rockenbauer and P. Tordo, J. Org. Chem., 2005, 70, 1198.

38 M. Matsushita, K. Kamata, K. Yamaguchi and N. Mizuno, J. Am. Chem. Soc., 2005, 127, 6632.

39 D. Moonshiram, J. W. Jurss, J. J. Concepcion, T. Zakharova, I. Alperovich, T. J. Meyer and Y. Pushkar, J. Am. Chem. Soc., 2012, 134, 4625.

40 G.-D. Lei and L. Kevan, J. Phys. Chem., 1991, 95, 4506.

41 H. Tsunoyamo, N. Ichikuni, H. Sakurai and T. Tsukuda, J. Am. Soc. Chem., 2009, 131, 7086.

42 M. D'Arienzo, J. Carbajo, A. Bahamonde, M. Crippa, S. Polizzi, R. Scotti, L. Wahba and F. Morazzoni, J. Am. Soc. Chem., 2011, 133, 17652.
43 T. Mitsudome, Y. Takahashi, T. Mizugaki, K. Jitsukawa and K. Kaneda, Angew. Chem., Int. Ed., 2014, 53, 8348.

44 J. Ohyama, T. Sato, Y. Yamamoto, S. Arai and A. Satsuma, J. Am. Chem. Soc., 2013, 135, 8016.

45 A. Lewera, W. P. Zhou, C. Vericat, J. H. Chung, R. Haasch, A. Wieckowski and P. S. Bagus, Electrochim. Acta, 2006, 51, 3950 .

46 V. Mazzieri, F. Coloma-Pascual, A. Arcoya, P. C. L'Argentière and N. S. Figoli, Appl. Surf. Sci., 2003, 210, 222.

47 R. Bavand, A. Yelon and E. Sacher, Appl. Surf. Sci., 2015, 355, 279.

48 R. Kotz, H. J. Lewerenz and S. Stucki, J. Electrochem. Soc., 1983, 130, 825.

49 Z. L. Liu, J. Y. Lee, M. Han, W. X. Chen and L. M. Gan, J. Mater. Chem., 2002, 12, 2453.

50 H. Hayashi, A. Ueda, A. Suino, K. Hiro and Y. Hakuta, J. Solid State Chem., 2009, 182, 2985.

51 S. A. Simakov and Y. Tsur, J. Nanopart. Res., 2007, 9, 403.

$52 \mathrm{~S} . \mathrm{Yu}, \mathrm{S}$. Li, X. Ge, M. Niu, H. Zhang, C. Xu and W. Li, Ind. Eng. Chem. Res., 2014, 53, 2238.

53 N. Lock, M. Bremholm, M. Christensen, J. Almer, Y.-S. Chen and B. B. Iversen, Chem.-Eur. J., 2009, 15, 13381. 